\section{Lack of spontaneous venous pulsation: possible risk indicator in normal tension glaucoma?}

\author{
Luís Abegão Pinto, ${ }^{1,2}$ Evelien Vandewalle, ${ }^{3}$ Eline De Clerck, ${ }^{3}$ \\ Carlos Marques-Neves ${ }^{2}$ and Ingeborg Stalmans ${ }^{3}$ \\ ${ }^{1}$ Department of Ophthalmology, Centro Hospitalar de Lisboa Central, Lisboa, \\ Portugal \\ ${ }^{2}$ Faculty of Medicine, Lisbon University, Lisbon, Portugal \\ ${ }^{3}$ Department of Ophthalmology, University Hospitals Leuven (UZ Leuven), Leuven, \\ Belgium
}

\begin{abstract}
.
Purpose: Recently, the absence of spontaneous venous pulsation (SVP) has been suggested as a vascular risk factor for primary open-angle glaucoma (POAG). As the mechanism behind this phenomenon is still unknown, the authors have studied this vascular component using colour Doppler imaging (CDI).

Methods: A total of 236 patients were divided into three diagnostic groups: healthy controls (81), POAG (86) and normal tension glaucoma (NTG; 69). All subjects were submitted to CDI studies of the retrobulbar circulation, intraocular pressure measurements and assessment of SVP existence. Mann-Whitney, chisquare contingency tables and Spearman correlations were used to explore differences and correlations between variables in the diagnostic groups.

Results: Eighty-two percent of healthy controls had SVP (66/81), while a smaller numbers were registered in both glaucoma groups: POAG - 50\% $(43 / 86)$; NTG $51 \%(35 / 69)$. In NTG patients, but not in POAG patients, the prevalence of the SVP phenomenon decreases with increased glaucoma damage $(p=0.04 ; p=0.55$, respectively). Overall glaucoma patients from both groups had lower central retinal vein $(C R V)$ velocities than the healthy controls $(p<0.05)$. NTG patients with SVP had less severe visual field defects (mean defect -6.92 versus $-11.1, p<0.05$ ), higher [correction added after online publication 21 September 2012; the word 'higher' has been inserted to replace the word 'lower'] peak systolic and mean flow velocities in the central retinal artery $(p<0.01 ; p<0.05$, respectively) as well as higher [correction added after online publication 21 September 2012; the word higher has been inserted to replace the word lower] maximal velocities and RI of the CRV (p < 0.02; p $<0.05$, respectively).

Conclusions: Glaucoma patients have a decrease in CRV velocities. SVP is less prevalent in glaucoma patients than in healthy individuals. This phenomenon apparently reflects different hemodynamic patterns in the central retinal vessels. This variable may be of particular importance in NTG patients, where it may be associated with more advanced functional damage.
\end{abstract}

Key words: colour Doppler imaging - glaucoma - normal tension glaucoma - spontaneous venous pulsation - vascular dysfunction

Acta Ophthalmol. 2013: 91: 514-520

(c) 2012 The Authors

Acta Ophthalmologica @ 2012 Acta Ophthalmologica Scandinavica Foundation

doi: $10.1111 /$ j.1755-3768.2012.02472.x

\section{Introduction}

Glaucoma is the second most common cause of blindness in the developed world (Kingman 2004). While intraocular pressure (IOP) lowering strategies have been the mainstay of glaucoma treatment, a significant number of patients still progress despite an otherwise successful reduction in this major risk factor (Leske et al. 2003; Parc et al. 2001). The vast majority of the studies regarding nonIOP-related risk factors such as vascular disturbances have focused on the arterial component. In fact, patients with primary open-angle glaucoma (POAG) but particularly the ones without increased IOP (normal tension glaucoma, NTG) have been found to have an altered systemic and ocular circulation, ranging from decreased ocular perfusion pressures and blood flow velocities (Stalmans et al. 2009; Garhöfer et al. 2010) to vascular endothelial dysfunction and defective autoregulation (Fadini et al. 2010; Flammer \& Mozaffarieh 2008; Resch et al. 2009a,b). However, glaucoma is also linked to venous diseases, being one of the main risk factors for central vein occlusion (The Eye Disease Case-Control Study Group 1996). Current evidence suggests this relation may be more important than previously thought, with a number of authors describing increased glaucoma 
progression in patients with increased venous pulse pressure (Morgan et al. 2005; Balaratnasingam et al. 2007). Evidence of this disturbance in the venous circulation is raising, ranging from increased episcleral venous pressure (Selbach et al. 2005), to a possible role in the appearance of optic disc haemorrhages (Krakau 1994). Additionally, glaucoma patients have a significant decrease in prevalence of the spontaneous venous pulsation (SVP) phenomenon over the optic disc, which can be found in up to $98 \%$ of the general population (Morgan et al. 2004; Legler \& Jonas 2009). Classically, this sign has been used to evaluate patients with papiledema, Graves' orbitopathy and as a prognostic sign in central vein occlusions (Jonas \& Harder 2003; Jonas 2003; Jonas 2004a,b). While the debate over the mechanisms involved in this absent pulsation is still ongoing, data suggest this may reflect an imbalance between the intra- and extraocular compartments pressure pulses (Donnelly \& Subramanian 2009; Nowroozzadeh \& Saki 2009; Kain et al. 2010). Interestingly, and despite the clinical relevance of this venous vascular component, there have been no studies on the ocular blood flow in these patients that can help determine whether this venous alteration is part of a larger vascular dysregulation. Colour Doppler imaging (CDI) technology has been a well-validated tool in ocular blood flow research (Stalmans et al. 2011; Founti et al. 2011), providing an increasing amount of relevant information to glaucoma studies, including on vascular dysregulation (Abegão Pinto et al. 2012).

Our study aimed to investigate differences in the retrobulbar circulation in glaucoma patients with and without a visible SVP phenomenon using the CDI technology.

\section{Methods}

\section{Subject groups}

Three cohorts of individuals over 18 years old were recruited for the study: patients with NTG $(n=69)$, patients with POAG $(n=86)$ and healthy control subjects $(n=81)$ of comparable age. This latter group was recruited from the persons accompanying the patients. Glaucoma patients were defined as having characteristic optic disc damage and visual field loss (Jamel 1997; Zeyen 1999). For the diagnosis of POAG, an untreated IOP of $21 \mathrm{mmHg}$ or greater was required. Current medical treatment, including topical IOL lowering drugs, was continued. The healthy volunteers were screened by a senior member of the glaucoma clinic (IST). Those with a family history of glaucoma, an increased or asymmetrical cup/disc ratio or any other optic disc structural change (notching, disc haemorrhage) or an IOP above $21 \mathrm{mmHg}$ were considered as possible glaucoma suspects and excluded. Patients with a history of ocular trauma or any known eye disease other than glaucoma were excluded. A history of ocular venous obstruction (branch or central occlusion) or systemic conditions associated with venous congestion (e.g. heart failure) was also considered to be exclusion criteria. Additionally, any optic disc abnormality or arteries' disposition that precluded the observation of the veins over the optic disc was also excluded.

Information regarding functional and structural damage from glaucoma patients was collected from examinations undertaken on the day of the study visit.

\section{Measuring devices}

Visual acuity was tested using the Early Treatment of Diabetic Retinopathy Study (ETDRS) chart. The chart was placed in the same location at the same distance from the patient under the same illumination for all subjects. IOP was measured with the Goldmann applanation tonometer (GAT). Blood pressure measurement was taken from subject's right arm using an electronic sphygmomanometer (Omron, Schaumburg, IL, USA). Retrobulbar flow velocities [peak systolic velocity (PSV), end diastolic velocity (EDV), mean flow velocity (MFV), maximum venous velocity (Vmax), minimum venous velocity (Vmin) and resistivity index (RI)] of the central retinal artery and vein [CRA and central retinal vein (CRV), respectively], nasal and temporal short posterior ciliary arteries (NPCA and TPCA, respectively) and ophthalmic artery (OA) were measured with the Antares CDI device (Siemens, Munich,
Germany). Observation of the optic disc was performed with a fundus camera (Topcon TRC-50DX/EX fundus camera; Topcon Medical Systems, Inc, Oakland, NJ, USA).

\section{Experimental design}

Patients were instructed to avoid caffeine intake, smoking and exercise for $3 \mathrm{hr}$ prior to the study visit. The study was approved by the ethical committee (Institutional Review Board) at the University Hospitals Leuven and was conducted in accordance with Good Clinical Practice within the tenets of the Helsinki agreement. Each patient/subject was required to sign an informed consent statement before being enrolled in the study and prior to any study measurements being taken. During the study visit, the following examinations were performed in the same order: visual acuity, IOP measurement by GAT, blood pressure and heart rate measurements, CDI and finally SVP assessment. This latter examination was performed using the following protocol: pharmacological dilation of the selected eye with tropicamide $\quad 0.5 \% \quad$ (Tropicol ${ }^{\circledR}, \quad$ Théa Pharma, Wetteren, Belgium), observation of the optic disc for at least 1 min using a fundus camera. Venous pulsations over the optic disc were described as either present/absent, and any asymmetry in superior/inferior pulsation was recorded. These last two examinations (CDI and SVP assessment) were performed by a single observer (LAP) masked to the patient diagnosis. Only one eye per patient was included in the study. The eye with greater glaucomatous damage was selected in the glaucoma patients and a randomly selected eye in the healthy individuals.

\section{Statistical analysis}

Chi-square tests (for $3 \times 2$ contingency tables) were used to analyse the spontaneous/absent venous pulsation ratios. Kruskal-Wallis tests were used to compare the three diagnostic groups on different variables. MannWhitney test was used in pairwise comparisons. The existence of correlation was explored using Spearman's correlation. Statistical significance was considered when $\mathrm{p}<0.05$. Values are depicted as mean \pm SD unless 
otherwise indicated. Analyses were performed using Graphpad Prism ${ }^{\circledR}$ ver. 5.0; (Graphpad Software Inc, La Jolla, CA, USA).

\section{Results}

\section{Patient's characteristics}

Table 1 summarizes the patient characteristics in the different diagnostic groups. Kruskall-Wallis test indicated no overall differences between the studied groups in age, blood pressure (BP) systolic and median ocular perfusion pressure (MOPP) ( $\mathrm{p}$ ranged from 0.13 to 0.91 ). The overall difference in diastolic BP that was detected between the three groups $(p<0.01)$ did not reach significance when comparing between the two glaucoma groups ( $p=0.12$ ) using a Mann-Whitney test. We identified statistically significant differences in visual acuity and IOP when comparing between the three groups $(\mathrm{p}<0.01$ ), which were judged clinically not unexpected nor relevant for the study. As expected, there was a significant difference in functional and structural parameters of glaucoma damage between the three groups (MD and RNFL thickness: $p<0.001)$. However, when comparing between the two glaucoma groups, no difference was detected (MD: $p=0.79 ; \quad$ RNFL thickness: $\mathrm{p}=0.90)$. A statistical significance between the SVP status in the three groups existed, with the healthy group having a higher prevalence of this phenomenon ( 66 of $81=82 \%$ ) when compared to both POAG (43 of $86=50 \%$ ) and NTG groups (35 of
$69=51 \%$. No difference was detected when comparing both glaucoma groups $(\mathrm{p}=0.75)$. Topical medications in both glaucoma SVP subgroups (with/without the SVP phenomenon) are summarized in Table 2.

\section{Experimental groups by SVP status}

Table 3 summarizes the analysis of the two subdivision within each experimental group according to their SVP status. In the healthy group, no differences were detected between patients that presented SVP to those who did not, regarding the ocular and systemic variables currently studied. In the POAG group, the only differences between the two sets of patients was a significantly higher MOPP and diastolic blood pressures $(p<0.01)$ in the patients who did not present SVP. The only statistically significant difference in the NTG group was a significantly higher functional damage in patients without SVP $(p<0.05)$ despite a similar degree of structural damage $(\mathrm{p}=0.68)$.

Despite an overall similarity in SVP prevalence in both POAG and NTG groups $(50 \%$ and $51 \%$, respectively; $\mathrm{p}=0.72$ ), further analysis of this prevalence according to the degree of glaucoma damage revealed differences between the two glaucoma groups (Fig. 1). In the NTG group, SVP prevalence decreased with increased functional damage, from $73 \%$ (11 of $15)$ in patients between +2 and $-2 \mathrm{~dB}$ to $56 \%$ (15 of 27$)$ in patients between -2 and $-10 \mathrm{~dB}$, and $33 \%$ (nine of 27 ) in patients with MD above $-10 \mathrm{~dB}$ $(p=0.04)$. In the POAG group, no differences in SVP prevalence were detected (MD between +2 and $-2 \mathrm{~dB}$ : 10 of $18=56 \%$; MD between -2 and $-10 \mathrm{~dB}: 15$ of $35=43 \%$; MD above $-10 \mathrm{~dB}$ : 18 of $33=55 \%$; $\mathrm{p}=0.55)$.

\section{Retrobulbar flow velocities in glaucoma patients and healthy controls}

The blood flow velocities obtained by CDI of the retrobulbar vessels (CRA, NPCA, TPCA, OA and CRV) of the overall groups and their statistical comparison are described in Table 4.

Table 5 depicts the data from the CDI examinations according to the SVP status of the three experimental groups. Healthy patients without SVP had a higher Vmin than their SVP positive counterpart $(p<0.05)$. POAG patients who did not show SVP, on the other hand, had lower CRV Vmin and RI than POAG patients with SVP ( $\mathrm{p}<0.02 ; \quad \mathrm{p}<0.05, \quad$ respectively). The NTG SVP-absent subgroup had significantly lower central retinal artery PSV and MFV ( $p<0.01 ; \mathrm{p}<0.05$, respectively), as well as lower Vmax and RI of the CRV $(\mathrm{p}<0.02$; $\mathrm{p}<0.05$, respectively) than the NTG patients with SVP phenomenon. No other differences were detected in any of the other pairwise comparisons $(\mathrm{p}>0.05)$.

\section{Discussion}

This study was conducted to identify characteristics that could be associated with the SVP phenomenon in glaucoma. Secondly, we checked whether any differences in such vari-

Table 1. Patients characteristics.

\begin{tabular}{|c|c|c|c|c|c|}
\hline & Healthy, $n(\%)$ & NTG, $n(\%)$ & POAG, $n(\%)$ & Overall (p-value) & Pairwise NTG versus POAG (p-value) \\
\hline$n$ & 81 & 69 & 86 & & \\
\hline Age & $64.6(14)$ & $69.3(11)$ & $67.4(12)$ & 0.13 & 0.45 \\
\hline IOP & $16.0(4.8)$ & $12.3(2.8)$ & $14.8(5.0)$ & $<0.001$ & $<0.001$ \\
\hline Visual acuity & $0.15(0.3)$ & $0.27(0.3)$ & $0.21(0.3)$ & $<0.01$ & 0.11 \\
\hline $\mathrm{SVP}+$ & $66(81)$ & $35(51)$ & $43(50)$ & $<0.001$ & 0.75 \\
\hline MD & $-0.6(3.9)$ & $-8.94(8.4)$ & $-9.3(8.6)$ & $<0.001$ & 0.79 \\
\hline RNFL thickness & $0.24(0.1)$ & $0.15(0.1)$ & $0.15(0.1)$ & $<0.001$ & 0.90 \\
\hline Pachymetry & $577(57)$ & $553(35)$ & $555(36)$ & 0.03 & 0.73 \\
\hline Systolic BP & $151(21)$ & $152(24)$ & $151(22)$ & 0.91 & 0.70 \\
\hline Diastolic BP & $81.8(12)$ & 84.3 (13) & 86.9 (12) & $<0.01$ & 0.12 \\
\hline MOPP & $55.5(9.9)$ & $58.9(9.3)$ & $57.4(10)$ & 0.16 & 0.34 \\
\hline
\end{tabular}

Mean values (and SD) are depicted. SVP refers to number of patients who presented that phenomenon compared to the total number of patients. Overall comparisons between the groups were made using the Kruskal-Wallis test. NTG versus POAG comparison was made using Mann-Whitney test. The differences in prevalence of SVP between the groups, however, were performed using a chi-square contingency table $(3 \times 2)$. SVP + indicates the presence of spontaneous venous pulsation; IOP $=$ intraocular pressure; $\mathrm{MD}=$ mean defect; RNFL $=$ retinal nerve fibre layer; $\mathrm{BP}=$ blood pressure; MOPP $=$ median ocular perfusion pressure $\left([2 / 3 \text { diastolic }+1 / 3 \text { systolic BPs }]^{*} 2 / 3-\right.$ Goldmann tonometry $)$. 
Table 2. Topical medications.

\begin{tabular}{llllll}
\hline & & $\begin{array}{l}\text { Beta-blockers, } \\
n(\%)\end{array}$ & $\begin{array}{l}\text { Alpha-agonists, } \\
n(\%)\end{array}$ & $\begin{array}{l}\text { Carbonic anhydrase } \\
\text { inhibitors, } n(\%)\end{array}$ & $\begin{array}{l}\text { Prostaglandin } \\
\text { analogs, } n(\%)\end{array}$ \\
\hline POAG & SVP + & $20(47)$ & $5(12)$ & $10(23)$ & $26(60)$ \\
& SVP- & $22(51)$ & $3(7)$ & $11(16)$ & $27(63)$ \\
NTG & SVP + & $14(40)$ & $3(9)$ & $12(34)$ & $12(34)$ \\
& SVP- & $12(35)$ & $3(9)$ & $12(35)$ & $16(47)$ \\
\hline
\end{tabular}

Number of patients and percentage (between brackets) are depicted. conditions of ischaemia and hypoxia, there is a known effect of hypertrophy and increased resistance of an otherwise very low resistance vascular bed (Takahashi et al. 2011). This process can even lead to an arterialization phenomenon of the vein to occur in such environment. In the ocular circulation, recent reports have been made

Table 3. Characteristics of the experimental groups according to SVP status.

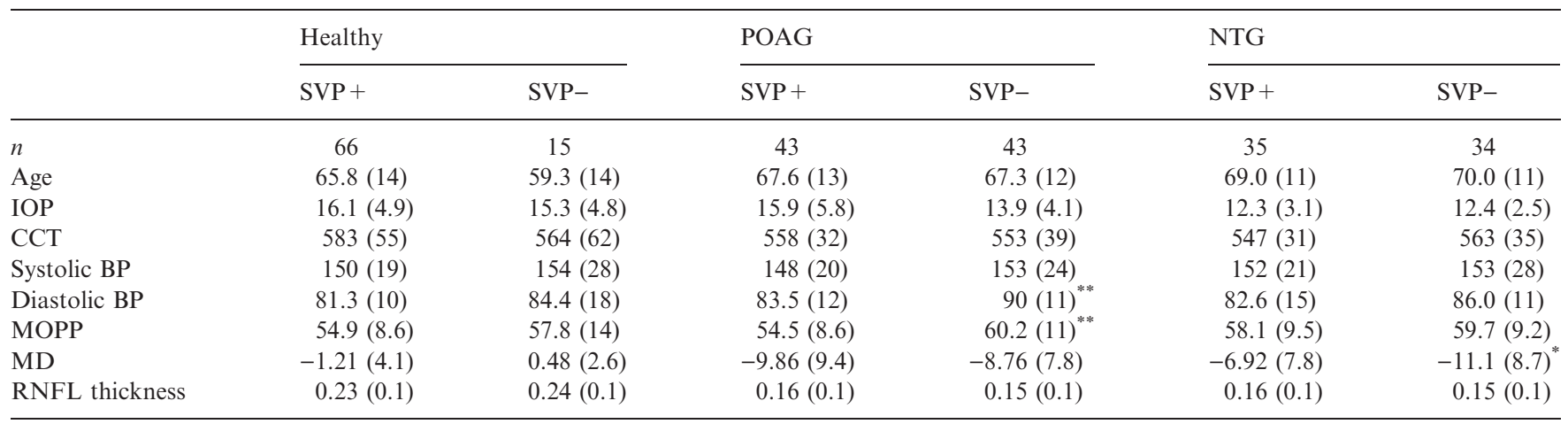

Data shown as mean (SD). Comparison between the subgroups was made using Mann-Whitney tests. *** Indicated statistically significant differences in pairwise comparison with SVP + counterpart $(\mathrm{p}<0.05 ; \mathrm{p}<0.01$, respectively). POAG $=$ primary open-angle glaucoma; NTG $=$ normal tension glaucoma; SVP = spontaneous venous pulsation; IOP = intraocular pressure; CCT $=$ central corneal thickness; $\mathrm{BP}=$ blood pressure; $\mathrm{MOPP}=$ mean ocular perfusion pressure $\left([2 / 3 \text { diastolic }+1 / 3 \text { systolic } \mathrm{BPs}]^{* 2 / 3-G o l d m a n n}\right.$ tonometry); $\mathrm{MD}=\mathrm{mean}$ defect; $\mathrm{RNFL}=$ retinal nerve fibre layer.

ables existed between NTG and POAG patients.

Both glaucoma populations presented a lower prevalence of the SVP phenomenon when compared to the healthy population. While this phenomenon has been previously described to occur in glaucoma (Morgan et al. 2004; Legler \& Jonas 2009),

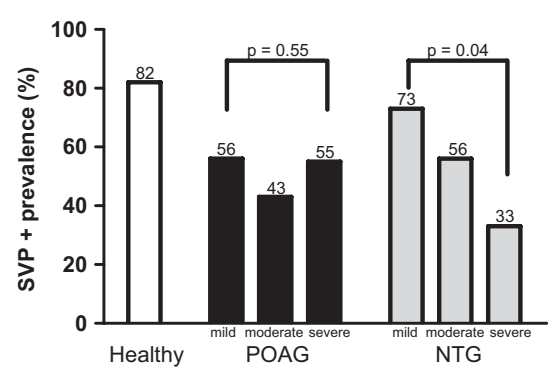

Fig. 1. Spontaneous venous pulsation prevalence in the three diagnostic groups. Glaucoma groups are divided into three categories according to increased functional damage criteria: mild - MD between +2 and $-2 \mathrm{~dB}$; moderate - MD between -2 and $-10 \mathrm{~dB}$; severe - MD above $-10 \mathrm{~dB}$. Values presented in percentage. p-Values represent overall comparison between each group categories. Statistical tests performed with chi-square contingency tables $(3 \times 2)$. no data existed concerning NTG patients specifically. However, there are significant differences in both glaucoma groups regarding this phenomenon. In NTG patients, the pulsating phenomenon prevalence decreased significantly with more advanced glaucomatous visual field damage, a relation we failed to detect in POAG patients. Our data suggest that the SVP phenomenon has a different meaning depending on the type of glaucoma. One possible explanation for this may be that the arterial vascular dysfunction, known to exist in glaucoma patients, also has an effect on the venous circulation. Endothelin, a well-known vasoconstrictor agent with a likely link to glaucoma (Shoshani et al. 2012), has a strong impact on the venous endothelium (Gao \& Raj 2005). Interestingly, it has been shown to be increased in patients with retinal vein occlusions (Iannaccone et al. 1998) as well as patients with both branch occlusions and NTG (Sin et al. 2011). In other vascular beds, such as in the lungs, veins have a nearly tenfold higher sensibility to endothelin than the corresponding arteries (Wang \& Coceani 1992). In about arterial-like endothelial receptors in the CRV in the postlamina cribosa segment (Kang et al. 2011). Several studies using endothelin receptor antagonists have detected an impact on retinal venous flow (Polak et al. 2003; Resch et al. 2009a,b).

Furthermore, as veins are structurally more fragile than their arterial counterparts, they are also more liable to mechanic compression. The glaucoma-associated deformation of the pores in the lamina cribosa might act as an outside compressing structure, thereby interfering in venous output and increasing vascular resistance, thus providing an additional mechanical explanation to the lack of pulsation.

The presumed increase in retinal venous pressure, implied in the absence of the pulsating phenomenon, has a direct consequence on the perfusion pressure. Calculations regarding ocular perfusion assume for practical reasons that retinal veins have similar values as IOP. Because in non-pulsating patients the retinal venous pressure is higher than IOP by an undetermined amount, the calculated MOPP is necessarily overestimated 
Table 4. CDI variables of the retrobulbar vessels in the experimental groups.

\begin{tabular}{lllcccl}
\hline & & Healthy cm/s (SD) & POAG cm/s $(\mathrm{SD})$ & NTG cm/s $(\mathrm{SD})$ & Overall p-value & Pairwise POAG versus NTG p-value \\
\hline CRA & PSV & $11.9(4.3)$ & $10.1(3.1)$ & $10.7(3.4)$ & 0.04 & 0.56 \\
& EDV & $3.20(1.2)$ & $2.70(0.9)$ & $2.96(1.1)$ & 0.01 & 0.11 \\
& RI & $0.72(0.1)$ & $0.73(0.1)$ & $0.71(0.1)$ & 0.70 & 0.43 \\
& MFV & $6.42(2.3)$ & $5.50(1.7)$ & $5.75(1.9)$ & 0.04 & 0.60 \\
CRV & Vmax & $5.89(1.6)$ & $5.13(1.3)$ & $5.32(1.5)$ & $<0.01$ & 0.47 \\
& Vmin & $3.40(0.8)$ & $3.09(0.6)$ & $3.08(0.7)$ & $<0.01$ & 0.42 \\
& RI & $0.41(0.1)$ & $0.38(1.0)$ & $0.41(0.1)$ & 0.08 & 0.07 \\
NPCA & PSV & $9.29(2.7)$ & $9.51(2.9)$ & $9.51(2.8)$ & 0.73 & 0.88 \\
& EDV & $3.13(1.1)$ & $3.15(1.0)$ & $3.2(1.0)$ & 0.77 & 0.65 \\
& RI & $0.66(0.1)$ & $0.70(0.4)$ & $0.66(0.1)$ & 0.84 & 0.69 \\
TPCA & MFV & $5.50(1.8)$ & $5.59(1.8)$ & $5.69(1.7)$ & 0.68 & 0.74 \\
& PSV & $8.97(2.4)$ & $9.90(1.8)$ & $9.20(2.7)$ & 0.06 & 0.09 \\
& EDV & $3.08(0.9)$ & $3.37(1.1)$ & $3.06(0.9)$ & 0.16 & 0.12 \\
& RI & $0.65(0.1)$ & $0.65(0.1)$ & $0.66(0.1)$ & 0.78 & 0.64 \\
OA & MFV & $5.40(1.4)$ & $6.00(1.8)$ & $5.44(1.5)$ & 0.06 & 0.06 \\
& PSV & $37.7(17)$ & $31.7(11)$ & $31.6(10)$ & 0.04 & 0.99 \\
& EDV & $6.85(3.9)$ & $5.93(3.0)$ & $5.81(3.7)$ & 0.26 & 0.53 \\
& RI & $0.82(0.1)$ & $0.81(0.1)$ & $0.82(0.1)$ & 0.52 & 0.27 \\
& MFV & $16.8(8.7)$ & $14.2(5.2)$ & $14.4(6.1)$ & 0.25 & 0.78
\end{tabular}

Data shown as mean velocities (SD). Overall comparison between the subgroups was made using Kruskal-Wallis test. Mann-Whitney tests were used in pairwise comparison. $\mathrm{NTG}=$ normal tension glaucoma; POAG = primary open-angle glaucoma; CRA = central retinal artery; $\mathrm{CRV}=$ central retinal vein; NPCA and TPCA $=$ short posterior ciliary arteries (nasal and temporal $=$ respectively); OA $=$ ophthalmic artery; $\mathrm{PSV}=$ peak systolic velocity; $\mathrm{EDV}=$ end diastolic velocity; RI = resistive index; MFV = mean flow velocity; Vmax = maximum velocity; Vmin $=$ minimum velocity

Table 5. CDI variables of the retrobulbar vessels according to SVP status.

\begin{tabular}{|c|c|c|c|c|c|c|c|}
\hline & & \multicolumn{2}{|l|}{ Healthy } & \multicolumn{2}{|l|}{ POAG } & \multicolumn{2}{|l|}{ NTG } \\
\hline & & $\begin{array}{l}\mathrm{SVP}+ \\
\mathrm{cm} / \mathrm{s}(\mathrm{SD})\end{array}$ & $\begin{array}{l}\mathrm{SVP}- \\
\mathrm{cm} / \mathrm{s}(\mathrm{SD})\end{array}$ & $\begin{array}{l}\mathrm{SVP}+ \\
\mathrm{cm} / \mathrm{s}(\mathrm{SD})\end{array}$ & $\begin{array}{l}\text { SVP- } \\
\mathrm{cm} / \mathrm{s}(\mathrm{SD})\end{array}$ & $\begin{array}{l}\mathrm{SVP}+ \\
\mathrm{cm} / \mathrm{s}(\mathrm{SD})\end{array}$ & $\begin{array}{l}\mathrm{SVP}- \\
\mathrm{cm} / \mathrm{s}(\mathrm{SD})\end{array}$ \\
\hline \multirow{4}{*}{ CRA } & PSV & $12.1(4.4)$ & $11.1(3.8)$ & $10.2(3.6)$ & $10.0(2.6)$ & $11.8(3.4)$ & $9.46(3.0)^{* * * *}$ \\
\hline & EDV & $3.21(1.1)$ & 3.14 (1.4) & $2.73(1.0)$ & $2.67(0.8)$ & $3.10(1.0)$ & $2.81(1.2)$ \\
\hline & RI & $0.72(0.1)$ & $0.73(0.1)$ & $0.72(0.1)$ & $0.72(0.1)$ & $0.73(0.1)$ & $0.70(0.1)$ \\
\hline & MFV & $6.45(2.3)$ & $6.23(2.5)$ & $5.52(2.0)$ & $5.47(1.4)$ & $6.19(1.8)$ & $5.27(1.9)^{*}$ \\
\hline \multirow[t]{3}{*}{ CRV } & $V \max$ & $5.77(1.5)$ & $6.54(1.9)$ & $5.31(1.3)$ & $4.94(1.4)$ & $5.68(1.7)$ & $4.78(0.9)^{* *}$ \\
\hline & Vmin & $3.32(0.7)$ & $3.84(1.1)^{*}$ & $3.07(0.6)$ & $2.67(0.83)^{* * *}$ & $3.13(0.7)$ & $2.90(0.4)$ \\
\hline & RI & $0.41(0.1)$ & $0.40(0.1)$ & $0.40(0.1)$ & $0.35(0.1)^{*}$ & $0.43(0.1)$ & $0.38(0.1)^{*}$ \\
\hline \multirow[t]{4}{*}{ NPCA } & PSV & $9.31(2.8)$ & $9.22(2.7)$ & $9.30(2.6)$ & $9.71(3.2)$ & $9.43(2.8)$ & $9.62(2.8)$ \\
\hline & EDV & $3.11(1.0)$ & $3.23(1.3)$ & $2.98(0.8)$ & $3.30(1.2)$ & $3.07(0.9)$ & $3.35(1.1)$ \\
\hline & RI & $0.66(0.1)$ & $0.65(0,1)$ & $0.67(0.1)$ & $0.66(0.1)$ & $0.66(0.1)$ & $0.65(0.1)$ \\
\hline & MFV & $5.46(1.8)$ & $5.83(1.7)$ & $5.31(1.6)$ & $5.85(2.0)$ & $5.52(1.5)$ & $5.88(1.9)$ \\
\hline \multirow[t]{4}{*}{ TPCA } & PSV & $8.80(2.5)$ & $9.75(2.0)$ & $10.2(3.1)$ & $9.64(2.4)$ & $9.44(2.7)$ & $8.93(2.6)$ \\
\hline & EDV & $3.01(0.8)$ & $3.42(1.1)$ & $3.27(0.9)$ & $3.47(1.2)$ & $3.01(0.8)$ & $3.06(0.9)$ \\
\hline & RI & $0.65(0.1)$ & $0.64(0.1)$ & $0.67(0.1)$ & $0.63(0.1)$ & $0.66(0.1)$ & $0.65(0.1)$ \\
\hline & MFV & $5.28(1.4)$ & 5.94 (1.4) & $6.02(1.8)$ & $5.98(1.8)$ & $5.54(1.5)$ & $5.33(1.5)$ \\
\hline \multirow[t]{4}{*}{ OA } & PSV & $38.5(17)$ & $33.1(9.9)$ & $32.0(12)$ & $31.6(8.8)$ & $31.2(10)$ & $32.1(11)$ \\
\hline & EDV & $7.04(4.0)$ & $5.86(3.6)$ & $5.34(2.7)$ & $6.44(3.2)$ & $5.30(2.3)$ & $6.34(4.7)$ \\
\hline & RI & $0.82(0.1)$ & $0.82(0.1)$ & $0.83(0.1)$ & $0.80(0.1)$ & $0.83(0.1)$ & $0.81(0.1)$ \\
\hline & MFV & $17.4(9.1)$ & $13.8(5.7)$ & $13.8(5.6)$ & $14.7(4.9)$ & $13.7(5.2)$ & $15.1(7.0)$ \\
\hline
\end{tabular}

Data shown as mean (SD) with velocities indicated as $\mathrm{cm} / \mathrm{s}$. Comparison between the subgroups was made using Mann-Whitney tests. ****** indicated statistically significant differences in pairwise comparison with $\mathrm{SVP}+$ counterpart $(\mathrm{p}<0.05$; $\mathrm{p}<0.02$; $\mathrm{p}<0.01$, respectively). SVP $=$ spontaneous venous pulsation; $\mathrm{NTG}=$ normal tension glaucoma; POAG $=$ primary open-angle glaucoma; CRA $=$ central retinal artery; CRV = central retinal vein; NPCA and TPCA short posterior ciliary arteries (nasal and temporal, respectively); OA = ophthalmic artery; $\mathrm{PSV}=$ peak systolic velocity; $\mathrm{EDV}=$ end diastolic velocity; $\mathrm{RI}=$ resistive index; $\mathrm{MFV}=$ mean flow velocity; $\mathrm{Vmax}=$ maximum velocity; Vmin minimum velocity.

(Morgan et al. 2004). As a risk factor for glaucoma progression (Leske 2009), there is a possibility that these patients have a much lower than mathematically expected perfusion pressure. Additionally, central retinal vessels had lower velocities in patients without venous pulsation. As decreased peak systolic velocities in the central retinal artery have been implicated in increased glaucoma progression (Zeitz et al. 2006), this com- 
bined arterial and venous decrease in ocular blood flow may signal an even wider vascular dysfunction.

Interestingly, the increased functional damage seen in non-pulsating NTG patient is not accompanied by a similar difference in structural damage assessment. The link between function-structure in glaucoma is still under debate and nonlinear relationships have been proposed (Hood \& Kardon 2007; Harwerth et al. 2010). Our results raise the possibility that the increase in venous pressure and consequent decrease in perfusion could lead to a metabolic impairment of the retinal cells, especially if associated with a local increase in vasoconstrictive agents. A consequent decrease in cellular activity could provide an additional explanation for this functional/structural mismatch. This hypothesis, however, needs to be verified by further studies on the metabolic activity of retinal cells in these patients.

Interestingly, POAG patients with an absent SVP phenomenon had higher diastolic pressures than their SVP positive counterparts. As diastolic pressure has been described as an independent risk factor for CRV occlusion (Arakawa et al. 2012), the lack of a visible SVP in POAG patients may, therefore, signal an increased risk for developing this retinal vascular disease. This hypothesis, however, needs to be confirmed by further studies.

Healthy patients without a SVP had higher minimal venous velocities. While the statistical significance of this finding has to be taken cautiously, this increase in Vmin without the consequent increase in Vmax could theoretically decrease venous pulsatility. Such decreases are seen in venous stasis conditions, such as lower limb vein or portal vein thrombosis (Hamper et al. 2007; Labropoulos et al. 2007).

Our work has several limitations. While the SVP phenomenon is clinically and easily assessed, the amount of additional venous retinal pressure implied by the non-pulsating vein remained undisclosed. Additionally, the venous output may have been altered by the ocular compression implied by tests used prior to the SVP assessment. While careful attention was paid not to exert pressure on the eye, the possibility of these measurements could have potentially interfered with the observation of this phenomenon has to be considered. However, because of the need of a mydriatic agent, SVP assessment was necessarily put in last place. One confounding factor that needs to be taken into account is that blood pressure medications were not discontinued. While blood pressure per se has been suggested not to be related to retinal venous pressure (Jonas 2004a,b), certain classes of medications can potentially interfere with the ocular venous tone. For instance, calcium channel blockers may block the vasoactive response of endothelin (Delgado et al. 2010).

Furthermore, the statistical analysis comparing the healthy subgroups should be considered carefully, as despite our series being by far the largest on the subject yet published, the study is still underpowered to fully address these questions. As such, any interpretation of both significant and nonsignificant $\mathrm{p}$ values of any comparison between healthy patients with and without SVP should be looked at cautiously.

In summary, our work suggests the easy to assess SVP phenomenon to be of clinical importance in glaucoma. Moreover, we demonstrate that glaucoma patients have decreased venous velocities in the CRV. While further studies on the venous circulation are still needed, this work emphasises that venous pulsations may be particularly important in NTG patients, where their absence may signal not only a wider vascular dysfunction but also a more advance stage of the disease.

\section{Acknowledgement}

The authors wish to thank Sien Boons and Veerle Vanbellinghen for their technical support.

\section{References}

Abegão Pinto L, Vandewalle E \& Stalmans I (2012): Disturbed correlation between arterial resistance and pulsatility in glaucoma patients. Acta Ophthalmol 90: e214-220.

Arakawa S, Yasuda M, Nagata M, Ninomiya T, Hirakawa $Y$, Doi $Y$, Kiyohara $Y$ \& Ishibashi T (2012): Nine-year incidence and risk factors for retinal vein occlusion in a general Japanese population: the Hisayama Study. Invest Ophthalmol Vis Sci 52: 59055909.

Balaratnasingam C, Morgan WH, Hazelton ML, House PH, Barry CJ, Chan H, Cringle SJ \& Yu DY (2007): Value of retinal vein pulsation characteristics in predicting increased optic disc excavation. Br J Ophthalmol 91: 441-444.

Delgado E, Marques-Neves C, Rocha I, Sales-Luís J \& Silva-Carvalho L (2010): Amlodipine effects on vasomotion in rabbit external ophthalmic artery. Graefes Arch Clin Exp Ophthalmol 248: 213-221.

Donnelly SJ \& Subramanian PS (2009): Relationship of intraocular pulse pressure and spontaneous venous pulsations. Am J Ophthalmol 147: 51-55.

Fadini GP, Pagano C, Baesso I et al. (2010): Reduced endothelial progenitor cells and brachial artery flow-mediated dilation as evidence of endothelial dysfunction in ocular hypertension and primary open-angle glaucoma. Acta Ophthalmol 88: 135-141.

Flammer J \& Mozaffarieh M (2008): Autoregulation, a balancing act between supply and demand. Can J Ophthalmol 43: 317321.

Founti P, Harris A, Papadopoulou D et al. (2011): Agreement among three examiners of colour Doppler imaging retrobulbar blood flow velocity measurements. Acta Ophthalmol 89: 631-634.

Gao Y \& Raj JU (2005): Role of veins in regulation of pulmonary circulation. Am J Physiol Lung Cell Mol Physiol 288: L213L226.

Garhöfer G, Fuchsjäger-Mayrl G, Vass C, Pemp B, Hommer A \& Schmetterer L (2010): Retrobulbar blood flow velocities in open angle glaucoma and their association with mean arterial blood pressure. Invest Ophthalmol Vis Sci 51: 6652-6657.

Hamper UM, DeJong MR \& Scoutt LM (2007): Ultrasound evaluation of the lower extremity veins. Radiol Clin North Am 45: 525-547.

Harwerth RS, Wheat JL, Fredette MJ \& Anderson DR (2010): Linking structure and function in glaucoma. Prog Retin Eye Res 29: 249-271.

Hood DC \& Kardon RH (2007): A framework for comparing structural and functional measures of glaucomatous damage. Prog Retin Eye Res 26: 688-710.

Iannaccone A, Letizia C, Pazzaglia S, Vingolo EM, Clemente G \& Pannarale MR (1998): Plasma endothelin-1 concentrations in patients with retinal vein occlusions. $\mathrm{Br}$ J Ophthalmol 82: 498-503.

Jamel H (1997): Target pressure in glaucoma therapy. J Glaucoma 6: 133-138.

Jonas JB (2003): Ophthalmodynamometric assessment of the central retinal vein collapse pressure in eyes with retinal vein stasis or occlusion. Graefes Arch Clin Exp Ophthalmol 241: 367-370.

Jonas JB (2004a): Ophthalmodynamometric determination of the central retinal vessel collapse pressure correlated with systemic 
blood pressure. Br J Ophthalmol 88: 501504

Jonas JB (2004b): Ophthalmodynamometric measurement of orbital tissue pressure in thyroid-associated orbitopathy. Acta Ophthalmol Scand 82: 239.

Jonas JB \& Harder B (2003): Ophthalmodynamometric estimation of cerebrospinal fluid pressure in pseudotumor cerebri. Br $\mathbf{J}$ Ophthalmol 87: 361-362.

Kain S, Morgan WH \& Yu DY (2010): New observations concerning the nature of central retinal vein pulsation. $\mathrm{Br} \mathrm{J}$ Ophthalmol 94: 854-857.

Kang MH, Balaratnasingam C, Yu PK, Morgan WH, McAllister IL, Cringle SJ \& Yu DY (2011): Morphometric characteristics of central retinal artery and vein endothelium in the normal human optic nerve head. Invest Ophthalmol Vis Sci 52: 1359-1367.

Kingman S (2004): Glaucoma is the second leading cause of blindness globally. Bull World Health Organ 82: 887-888.

Krakau CE (1994): Disk hemorrhages and retinal vein occlusions in glaucoma. Surv Ophthalmol 38: 18-21.

Labropoulos N, Borge M, Pierce K \& Pappas PJ (2007): Criteria for defining significant central vein stenosis with duplex ultrasound. J Vasc Surg 46: 101-107.

Legler U \& Jonas JB (2009): Frequency of spontaneous pulsations of the central retinal vein in glaucoma. J Glaucoma 18: 210 212

Leske MC (2009): Ocular perfusion pressure and glaucoma: clinical trial and epidemiologic findings. Curr Opin Ophthalmol 20: 73-78.

Leske MC, Heijl A, Hussein M, Bengtsson B, Hyman L, Komaroff E \& Group Early Manifest Glaucoma Trial (2003): Factors for glaucoma progression and the effect of treatment: the early manifest glaucoma trial. Arch Ophthalmol 121: 48-56.

Morgan WH, Hazelton ML, Azar SL, House PH, Yu DY, Cringle SJ \& Balaratnasingam C (2004): Retinal venous pulsation in glau- coma and glaucoma suspects. Ophthalmology 111: 1489-1494.

Morgan WH, Balaratnasingam C, Hazelton ML, House PH, Cringle SJ \& Yu DY (2005): The force required to induce hemivein pulsation is associated with the site of maximal field loss in glaucoma. Invest Ophthalmol Vis Sci 46: 1307-1312.

Nowroozzadeh MH \& Saki S (2009): Intraocular pulse pressure affects the sensitivity and specificity of spontaneous venous pulsation test. Am J Ophthalmol 147: 945.

Parc CE, Johnson DH, Oliver JE, Hattenhauer MG \& Hodge DO (2001): The long-term outcome of glaucoma filtration surgery. Am J Ophthalmol 132: 27-35.

Polak K, Luksch A, Frank B, Jandrasits K, Polska E \& Schmetterer L (2003): Regulation of human retinal blood flow by endothelin-1. Exp Eye Res 76: 633-640.

Resch H, Garhofer G, Fuchsjäger-Mayrl G, Hommer A \& Schmetterer L (2009a): Endothelial dysfunction in glaucoma. Acta Ophthalmol 87: 4-12.

Resch H, Karl K, Weigert G, Wolzt M, Hommer A, Schmetterer L \& Garhöfer G (2009b): Effect of dual endothelin receptor blockade on ocular blood flow in patients with glaucoma and healthy subjects. Invest Ophthalmol Vis Sci 50: $358-363$.

Selbach JM, Posielek K, Steuhl KP \& Kremmer S (2005): Episcleral venous pressure in untreated primary open-angle and normaltension glaucoma. Ophthalmologica 219: 357-361.

Shoshani YZ, Harris A, Shoja MM, Rusia D, Siesky B, Arieli Y \& Wirostko B (2012): Endothelin and its suspected role in the pathogenesis and possible treatment of glaucoma. Curr Eye Res 37: 1-11.

Sin BH, Song BJ \& Park SP (2011): Aqueous vascular endothelial growth factor and endothelin-1 levels in branch retinal vein occlusion associated with normal tension glaucoma. J Glaucoma [Epub ahead of print].

Stalmans I, Harris A, Fieuws S, Zeyen T, Vanbellinghen V, McCranor L \& Siesky B
(2009): Color Doppler imaging and ocular pulse amplitude in glaucomatous and healthy eyes. Eur J Ophthalmol 19: 580 587.

Stalmans I, Vandewalle E, Anderson DR et al. (2011): Use of colour Doppler imaging in ocular blood flow research. Acta Ophthalmol 89: 609-630.

Takahashi H, Soma S, Muramatsu M, Oka M \& Fukuchi Y (2011): Upregulation of ET-1 and its receptors and remodeling in small pulmonary veins under hypoxic conditions. Am J Physiol Lung Cell Mol Physiol 280: L1104-L1114.

The Eye Disease Case-Control Study Group (1996): Risk factors for central retinal vein occlusion. Arch Ophthalmol 114: 545-554.

Wang Y \& Coceani F (1992): Isolated pulmonary resistance vessels from fetal lambs. Contractile behavior and responses to indomethacin and endothelin-1. Circ Res 71: $320-330$.

Zeitz O, Galambos P, Wagenfeld $\mathrm{L}$ et al. (2006): Glaucoma progression is associated with decreased blood flow velocities in the short posterior ciliary artery. Br J Ophthalmol 90: 1245-1248.

Zeyen T (1999): Target pressures in glaucoma. Bull Soc Belge Ophtalmol 274: 6165.

Received on March 5th, 2012.

Accepted on April 15th, 2012.

\section{Correspondence:}

Ingeborg Stalmans, MD, $\mathrm{PhD}$

Department of Ophthalmology

University Hospitals Leuven

Campus St Raphael, Kapucijnenvoer 33

B-3000 Leuven

Belgium

Tel: + 3216332372

Fax: + 3216332367

Email: ingeborg.stalmans@uzleuven.be 